Running head: MEMORY CONTEXTUALIZATION AND INTRUSIONS

Visuospatial context learning and configuration learning is associated with analogue traumatic intrusions

Journal of Behavior Therapy and Experimental Psychopathology, 54

Thomas Meyer* (a, d), Julie Krans (b), Vanessa van Ast (c), \& Tom Smeets (d)

(a) Behavioural Science Institute, Radboud University Nijmegen, The Netherlands

(b) Behavior, Health and Psychopathology, KU Leuven, Belgium

(c) Clinical Psychology, University of Amsterdam, The Netherlands

(d) Clinical Psychological Science, Maastricht University, The Netherlands

\footnotetext{
Author Note

* Correspondence concerning this article should be addressed to Thomas Meyer. Office phone: 0031-243612667. Office fax: 0031-243615937. E-mail address: t.meyer@ bsi.ru.nl.
} 


\begin{abstract}
Background and objectives: Cognitive and information processing theories of PostTraumatic Stress Disorder (PTSD) assert that trauma intrusions are characterized by poor contextual embedding of visuospatial memories. Therefore, efficient encoding of visuospatial contextual information might protect against intrusions. We tested this idea using indices of visuospatial memory embedding along with the trauma film paradigm.
\end{abstract}

Methods: Individual differences in spatial configuration learning, as well as the degree to which visual recognition memory depends on its visual encoding context (i.e., memory contextualization), were assessed in 81 healthy participants. Next, participants viewed a distressing film. Intrusions and other PTSD analogue symptoms were assessed subsequently.

Results: Participants displaying stronger memory contextualization developed fewer intrusions and PTSD analogue symptoms. Spatial configuration learning was unrelated to memory contextualization and, contrary to prior findings, predicted higher levels of intrusions.

Limitations: Due to the analogue design, our findings may not translate directly to clinical populations. Furthermore, due to the correlational design of the study, causal relations remain to be tested.

Conclusions: Our results suggest a protective role for the ability to integrate memories in their original visual learning context against the development of PTSD symptoms.

Key-words: Post-Traumatic Stress Disorder; Trauma Film Paradigm; Intrusions; Contextual memory; Spatial Contextual Cueing Task 


\section{Visuospatial context learning and configuration learning is associated with analogue traumatic intrusions}

Many people experience potentially traumatic situations at some point in their life, such as natural disasters, severe accidents, or violent crimes (Darves-Bornoz et al., 2008). Although most trauma victims adjust well (Bonanno \& Mancini, 2008), a considerable proportion develops trauma-related psychopathology, such as Post-Traumatic Stress Disorder (PTSD; American Psychiatric Association, 2013). Characteristically, many victims suffer from recurrent involuntary memories in the form of vivid images that are highly distressing and rich in sensory detail (e.g., intrusions, flashbacks). To explain the hyper-accessibility of traumatic memories, various theoretical models of PTSD attribute a critical role to mechanisms of memory encoding, consolidation, or retrieval (Brewin, Dalgleish, \& Joseph, 1996; Ehlers \& Clark, 2000; Rubin, Berntsen, \& Bohni, 2008).

Cognitive and information processing theories assert that intrusions in PTSD lack contextual integration within the autobiographical memory structure. For example, according to the revised dual-representation model (Brewin, Gregory, Lipton, \& Burgess, 2010), if representations of a traumatic experience have been stored in isolation from the original encoding context, matching perceptual cues can involuntarily trigger sensation-near representations, resulting in subjective re-experiencing of the trauma. Similarly, Ehlers and Clark (2000) posit that due to strong perceptual priming in PTSD, cues with even vague sensory similarity to the traumatic event can trigger intrusions, which often appear to be disjointed from relevant contextual information. Taken together, it follows that individuals who are better able to store and discriminate environmental cues from the original traumatic scenes might be less vulnerable to develop intrusive trauma memories.

In line with this assertion, Meyer, Smeets, Giesbrecht, Quaedflieg, Girardelli, et al. (2013) found that individuals who displayed superior implicit learning of spatial 
configurations reported fewer intrusive memories after viewing a trauma film. Arguably, individuals who excel in configuration learning are better able to distinguish trauma cues from perceptually similar stimuli in the environment, resulting in a lower likelihood of trauma memories being activated. Moreover, implicit spatial configuration learning depends crucially on medial temporal lobe structures (Goldfarb, Chun, \& Phelps, 2016; Preston \& Gabrieli, 2008) that have been theorized to be involved in the construction of abstract representations from visuospatial input (e.g., allocentric, or context-based representations; Brewin et al., 2010). Therefore, better spatial configuration learning might also be linked to efficient encoding and binding (also see Farrell, 2012) of the visuospatial context in which a traumatic event took place.

However, the ability to merely discriminate cues is necessary but perhaps not sufficient to protect against intrusions: central perceptual elements of a traumatic experience should also be integrated within the visual representation of their original encoding context (i.e., memory contextualization). Thereby, perceptual trauma memories would become more contextdependent and less likely to be triggered out of context. By including an additional novel task to assess memory contextualization, the current study investigated whether the association between spatial configuration learning and intrusions is mediated by memory contextualization.

We expected to replicate the finding that participants with superior spatial configuration learning report fewer intrusive memories after viewing a trauma film. Moreover, we predicted memory contextualization to be associated with fewer intrusive trauma film intrusions, because it may prevent emotional memories from being activated in isolation (Brewin et al., 2010; Ehlers \& Clark, 2000) and limit generalization of potential memory triggers. Finally, assuming that spatial configuration learning facilitates visuospatial scene discrimination, we expected this memory function to be associated with memory contextualization. 


\section{Method}

\section{Participants}

Eighty-one undergraduates (63 women) with a mean age of 21.5 years $(S D=2.7)$ were recruited via advertisements at Maastricht University campus and completed the study. Prior to the study, candidate participants received information about the study and a screening form via email. Candidates were instructed to reply only if they were still interested and eligible for participation, using the following exclusion criteria: (1) recent psychological or psychiatric problems, (2) drug or alcohol abuse, (3) blood phobia or a history of a severe accident, assault, or injury, and (4) prior participation in similar trauma film studies. Furthermore, participants were required to be fluent in Dutch and have normal or corrected-to-normal vision. They received partial course credit or financial compensation $(€ 25)$ in return for their participation. The study was approved by the standing ethical committee of the Faculty of Psychology and Neuroscience, Maastricht University.

\section{Spatial configuration learning}

We employed the abbreviated Spatial Contextual Cueing Task (SCCT; Bennett, Barnes, Howard, \& Howard, 2009; Meyer et al., 2013). Participants are required to find a single target with the shape of a rotated ' $\mathrm{T}$ ' pointing left or right among 11 ' $\mathrm{L}$ '-shaped distracters and indicate in which direction the target points as fast and accurately as possible by pressing a button. Across 30 blocks with 12 trials each, half of the target and distracter configurations were repeated, whereas novel configurations were presented on the other half of the trials. Target direction (left or right) varied randomly across all trials. Since repeated configurations predict the target location, they facilitate the search and reduce reaction times (RT) in repeated compared to novel configurations. This learning effect quantifies spatial configuration learning. For further details about the programming of this task, see Meyer et al. (2013; supplementary material). 
Scoring. The median RT of trials with accurate responses was extracted for each block, separately for novel and repeated trials. In line with Meyer et al. (2013), these scores were averaged across three consecutive epochs of 9, 10, and 10 blocks (omitting the very first block, because no repetition had occurred yet). To quantify spatial configuration learning, we subtracted average median RT scores on repeated trials from novel trials. A more positive difference score indicates stronger spatial configuration learning. In line with prior studies using the SCCT in healthy young adults (Bennett et al., 2009; Meyer et al. 2013), accuracy scores were close to ceiling $(M>95 \%)$ and therefore not considered in the analyses.

\section{Visual memory contextualization}

On the basis of a contextual memory task with word stimuli used previously by Van Ast, Cornelisse, Meeter, Joëls, and Kindt (2013), this task measures the speed and accuracy with which participants recognize neutral and emotional pictures of objects that were presented in an earlier encoding phase.

Stimuli. One-hundred and twenty pictures of different objects served as stimuli. The pictures were selected from the International Affective Picture System (IAPS; Lang, Bradley, \& Cuthbert, 2005) and their visual backgrounds were deleted using Photoshop (Adobe, San Jose, CA, USA). Objects were either neutral or negative. Within each valence category, there were equal numbers of objects most likely encountered indoors (e.g., chair, burglar), in nature (e.g., mushroom, snake), or in an urban context (e.g., umbrella, handgun). In addition, 60 creative-commons licensed pictures taken from the Internet were used, displaying emotionally neutral background scenes indoor (e.g., living room, $n=20$ ), nature (e.g., forest, $n=20$ ), or urban (e.g., city street, $n=20)$.

Encoding phase. Each trial began with a $1 \mathrm{~s}$ fixation cross on a black background, followed by a $700 \times 933$ pixels background scene picture that was shown for $2.5 \mathrm{~s}$. One second after background picture onset, an object picture appeared in the foreground within a 
grey 300 x 400 pixels frame. The object was displayed for $2 \mathrm{~s}$, outlasting background picture offset for $500 \mathrm{~ms}$. After that, participants were asked to provide valence and arousal ratings of the object within its background using two respective Self-Assessment Manikin (SAM; Bradley \& Lang, 1999) ratings in randomized order, in order to stimulate deep encoding (Craik \& Lockhart, 1972).

The encoding phase consisted of 60 trials, where each background picture was paired with one out of 60 object pictures (30 neutral / 30 negative). Trial sequence and stimulus composition was randomized for each participant, with the restriction that background-object combinations matched in terms of scene (i.e., indoor, nature, urban) and that the same valence (neutral, negative) did not occur more than 3 times consecutively. To reduce possible primacy effects, the task began with three additional, fixed practice trials with neutral objects. Participants were instructed to vividly imagine each foreground item and its meaning within the shown background scene, stimulating active binding of the object and background, rather than encoding of the object-background combination as a single compound (Staresina \& Davachi, 2010) ${ }^{1}$.

Recognition phase. Recognition trials began with a $1 \mathrm{~s}$ fixation cross, followed by presentation of a background picture. A foreground picture appeared $1 \mathrm{~s}$ after background picture onset. Simultaneously, the response options old and new appeared in the lower left and lower right corners of the background picture, requiring participants to indicate whether they recognized the object by pressing the left (' $z$ ') or right ('/') response key with the respective index finger. 'Old' and ' $n e w$ ' responses were randomly assigned to the left or right response key for each participant. Upon response, the next trial began.

The recognition phase consisted of 120 trials, whereby 60 trials with old objects were intermixed with 60 trials with new objects (30 neutral, 30 negative). In half of the trials with old objects, the object-background combination was the same as during encoding ('intact'). 
For the other 30 old object trials, the remaining background pictures were recombined to form new object-background pairs ('rearranged'), again assuring that objects and backgrounds matched in terms of scene. New objects were each paired with one of the 60 background pictures. The trial sequence was randomized individually, whereby trial type (old, new) and valence (neutral, negative) occurred no more than 3 times consecutively, and the same background picture could not occur twice within 4 trials.

Participants were instructed to indicate whether they had seen the depicted object during encoding by pressing the 'old' or 'new' button. They were told that the background-object combination could sometimes be the same as during encoding and sometimes not, but that they should focus only on the object and to respond as soon as they were sure about the correct answer. Accuracy and reaction time (RT) with respect to object picture onset were recorded for each trial. Three fixed practice trials were inserted at the beginning. Figure 1 displays exemplary stimuli of the contextual memory task and the possible background-object combinations during recognition.

--- insert Figure 1 about here ---

Scoring. Following signal detection theory, the $d$ ' sensitivity index was established by subtracting the $Z$-transformed false alarm rates from $Z$-transformed hit rates, separately for trials with neutral and negative stimuli. Perfect hit or false alarm rates were replaced with .975 and .025 , respectively (Stanislaw \& Todorov, 1999). To quantify the context dependence, $d$ ' sensitivity for rearranged trials was subtracted from intact trials. A more positive difference score indicates relatively better discrimination performance in trials with intact objectbackground combination, and thus, stronger memory contextualization.

For RT analyses, only accurate responses within $3 S D$ around the individual RT mean were included. Median RT scores were extracted for each valence (neutral, negative) and recognition type (old-intact, old-rearranged). Finally, memory contextualization was 
quantified by calculating the difference in median RT between intact and rearranged trials, separately for neutral and negative trials. To reduce the impact of individual differences in overall RT, we calculated the percentage difference relative to overall RT, i.e., [(Rearranged Intact $\left.)^{*} 100\right] /[($ Rearranged + Intact $) / 2]$. Thus, a more positive difference percentage reflects relatively faster recognition in trials with an intact object-background combination, and thus a larger benefit from the visuospatial context during recognition.

\section{Trauma film}

Participants viewed a 14 min compilation of aversive film fragments that was used in Meyer, Smeets, Giesbrecht, Quaedflieg, Girardelli, et al. (2013). Specifically, it consisted of graphic scenes of an eye and a knee surgery, a person drowning, a fatal car accident, and documented atrocities from the 1994 genocide in Rwanda. The compilation was comparable to, and partially overlapped with the one used previously by Holmes, James, Coode-Bate, and Deeprose (2009). Additional fragments were collected from different sources, including the road education film COW (co-produced by Gwent Police; www.gwent.police.uk), the documentary Ghosts of Rwanda (Artwork PBS, 2004), and clips retrieved from the internet (YouTube, www.youtube.com). For more details, please contact the corresponding author.

\section{Trauma film intrusions and PTSD analogue symptoms}

Participants were provided with a one-week diary (Holmes et al., 2009; translated to Dutch) in which they recorded their intrusions, defined as spontaneous, involuntary memories of the film. Participants were instructed to record intrusions as soon as they occurred, or the absence of intrusions at least twice a day. For each intrusion, they were asked to indicate the content (for verification that the intrusion was film-related) and trigger (if known), whether it consisted of images, thoughts, or both, and how distressing it was on an eleven-point scale (range: $0=$ not at all to $10=$ extremely). The frequencies of intrusions were log-transformed prior to the analyses to correct their right-skewed distribution $(\ln [1+\#$ memories $]$; for 
readability, we provide uncorrected descriptive statistics). Next to the overall intrusion frequency, our analyses also addressed the frequencies of intrusions with an image component and those with a thought component separately. Intrusions that had both components were assigned to both categories. In addition, mean distress scores were calculated across all recorded memories (distress scores were set to zero if no intrusions had occurred).

The Impact of Event Scale (IES; Horowitz, Wilner, \& Alvares, 1979) served as a retrospective measure of intrusion-related distress and overall PTSD analogue symptoms at one-week follow-up. The instructions were adapted to specifically measure symptoms related to the trauma film. The IES consists in total of 15 items $(\alpha=.81)$ that require respondents to indicate the frequency of specific symptoms on a four-point scale. The Intrusion Symptoms subscale ( 7 items, $\alpha=.73$ ) was of particular interest, while the total score (summarizing the intrusion and avoidance subscales) served as a measure of overall PTSD symptoms.

\section{Mood responses}

In order to measure emotional responding to viewing the trauma film, we used the state version of the Positive and Negative Affect Schedule (PANAS; Watson, Clark, \& Tellegen, 1988), consisting of a 10-items subscale for positive affect (PA) and one for negative affect (NA), all $\alpha \mathrm{s}>$.73. In addition, changes in the emotions 'fearful', 'shocked', 'sad', and 'angry' were assessed by means of four visual analogue scales (VAS) ranging from $0=$ not at all to $100=$ very much.

\section{Baseline psychopathology}

The 21-item version of the Depression Anxiety Stress Scale (DASS-21; Lovibond \& Lovibond, 1995) was used as a baseline measure of common symptoms of psychopathology. Its items present statements representing depression, anxiety, and stress-related symptoms, and respondents indicate whether these applied to them in the past week using 4-point scales 
(1=did not apply to me at all; 4=applied to me very much, or most of the time). We used the sum score of all items $(\alpha=.88)$ as a measure of general psychological distress.

\section{Procedure}

Participants were scheduled to two laboratory sessions separated by a one-week interval. They were informed beforehand that the materials used in the study could cause transient negative emotions and intrusions, and provided written informed consent. In session 1, participants were seated in front of a 22-inch widescreen monitor and completed the DASS-21 (all questionnaires were computer-administered). They then completed the encoding phase of the contextual memory task. The recognition phase took place $35 \mathrm{~min}$ after the end of the encoding phase. During this interval participants completed the SCCT, whereby they placed their head on a chin rest positioned $60 \mathrm{~cm}$ from the screen. After completion of these tasks, participants viewed the trauma film fragments. Before and after film viewing, participants completed the emotion VAS and the PANAS. During film viewing, participants were instructed to move as little as possible and to imagine being a witness or bystander to the depicted scenes, a procedure thought to enhance intrusion development (Hagenaars, van Minnen, Holmes, Brewin, \& Hoogduin, 2008). To further enhance immobility and immersion, and to reduce distraction, they were provided with headphones and were asked to place their head on the chin rest at $60 \mathrm{~cm}$ distance from the screen. An experimenter remained in the room, and in exceptional cases, reminded the participant to return their full attention to the film fragments. Afterwards, participants were given the diary and received extensive oral and written instructions on how to complete it. In the second laboratory session, the experimenter collected the diary and briefly discussed all entries (e.g., to resolve ambiguity due to poor handwriting), and filled out the IES. When the study was completed, participants were fully debriefed by email. We encouraged participants to ask any questions they might have at any 
time during or after the study, or to consult a trained psychologist whose contact details were provided (no participant made use of this option or reported continued distress to us).

\section{Statistical Analysis}

Single extreme scores in the distributions of the memory contextualization task and in spatial configuration learning scores were replaced so that their deviance from the sample mean equalled 2.5 times the sample $S D$ (i.e.,Winsorizing; Rivest, 1994). The main hypotheses were addressed with Pearson product-moment correlations. Within-subject effects were tested with paired samples $t$-tests and repeated measures ANOVAs. When sphericity assumptions were violated, Greenhouse-Geisser corrected $p$-values are reported, along with the respective Epsilon and uncorrected degrees of freedom. Alpha was set at .05 (two-tailed) for all tests.

\section{Results}

\section{Responses to the trauma film}

In response to film viewing, mean NA increased from 13.8 points $(S D=4.4)$ at pre-film to $19.0(S D=6.9)$ at post-film, $t(80)=7.64, p<.001$, whereas PA remained unchanged from pre$(M=23.1, S D=6.0)$ to post-film $(M=22.2, S D=5.0), t(80)=1.38, p=.17$. The VAS for the emotions anxious, shocked, angry, and sad similarly displayed significant increases, all $t \mathrm{~s}>6.17, p s<.001$, mean changes lying between $15.5(S D=20.1)$ for feeling anxious and 28.9 $(S D=25.1)$ for feeling shocked. Table 1 summarizes the PTSD analogue symptoms reported by our participants ${ }^{2}$.

--- insert Table 1 about here ---

\section{Spatial configuration learning and PTSD analogue symptoms}

Spatial configuration learning. A 2 (Configuration: novel, repeated) by 3 (Epoch: 1, 2, 3) repeated measures ANOVA revealed the learning effects that are typical for the SCCT. That is, participants became faster with time (Epoch main effect), $F(2,160)=96.98, \varepsilon=.86$, $p<.001, \eta p^{2}=.55$, and responded faster on repeated $(M=1835.0 \mathrm{~ms}, S E=49.3)$ compared to 
novel trials $(M=2049.5 \mathrm{~ms}, S E=42.7), F(1,80)=31.70, p<.001, \eta p^{2}=.28$. There also was a significant Configuration by Epoch interaction, $F(2,160)=23.97, \varepsilon=.82, p<.001, \eta p^{2}=.23$, indicating that the relative RT advantage for repeated configurations increased with time. As in Meyer, Smeets, Giesbrecht, Quaedflieg, Girardelli, et al. (2013; see supplemental materials), for the following correlation analyses, we used the difference score between repeated and novel configurations from the first epoch only, since these most likely reflect individual differences in spatial configuration learning ${ }^{3}$.

Correlations with PTSD analogue symptoms. In contrast to our expectations, spatial configuration learning scores correlated positively with the number of reported intrusions, $r=.32, p=.004$. This effect was largely carried by intrusions with an image component, $r=.33$, $p=.002$, and not statistically significant for thought-based intrusions, $r=.18, p=.11$.

Meanwhile, there was no association with the mean intrusion distress scores, $r=.10, p=.36$. On the IES, spatial configuration learning scores correlated positively with higher overall PTSD analogue symptoms, $r=.23, p=.042$. A significant correlation emerged for the intrusions subscale, $r=.32, p=.004$, but not for the avoidance subscale, $r=.08, p=.47$.

\section{Visual memory contextualization and PTSD analogue symptoms}

Memory contextualization. On average, participants were good at discriminating old from new objects in the visual recognition memory task, resulting in average $d$ ' sensitivity scores above 2.5 in all conditions (range: $0.7-3.92$ ). A 2 (Context: intact, rearranged) by 2 (Valence: neutral, negative) repeated measures ANOVA revealed the expected main effect of context, $F(1,80)=10.38, p=.002, \eta p^{2}=.12$, with higher discrimination performance in intact compared to rearranged contexts $\left(M_{\text {Difference }}=0.14, S E=0.04\right)$. There were no main or interaction effects involving valence, $F \mathrm{~s}<1.70, p \mathrm{~s}>.19, \eta p^{2}<.02$.

For RT, a 2 (Context: intact, rearranged) by 2 (Valence: neutral, negative) repeated measures ANOVA revealed a significant main effect of context, $F(1,80)=17.51, p<.001$, 
$\eta p^{2}=.18$. As expected, RT was shorter in intact as compared with rearranged trials

$\left(M_{\text {Difference }}=63.1 \mathrm{~ms}, S E=15.1\right)$. There also was a main effect of valence, $F(1,80)=18.21$, $p<.001, \eta p^{2}=.19$, with shorter RT in neutral trials than in negative trials $\left(M_{\text {Difference }}=99.9 \mathrm{~ms}\right.$, $S E=23.4)$. There was no interaction between valence and context, $F(1,80)<0.1, p=.90$.

Correlations with PTSD analogue symptoms. Since memory contextualization did not interact with valence for neither $d$ ' nor RT, we collapsed the difference scores between intact and rearranged trials across neutral and negative per participant for both $d$ ' and RT. For the $d$ ' memory contextualization score, all correlations with PTSD analogue symptoms were smaller than $r=-.13$ and not statistically significant (all $p \mathrm{~s}>.28$ ).

Notably, $d$ ' memory contextualization scores were also unrelated to RT memory contextualization, $r=.03, p=.76$, suggesting that accuracy of memory contextualization was not related to speed of responding. RT memory contextualization scores correlated negatively with the number of intrusions $(r=-.33, p=.003)$, consistently for both image-based $(r=-.30)$ and thought-based ( $r=-.34)$ intrusions (see Figure 2). Similar associations were found for the mean distress scores across all reported intrusions, $(r=-.31, p=.005)$. Moreover, higher RT contextualization scores correlated with fewer PTSD analogue symptoms on the IES, $(r=-.24$, $p=.03)$. This effect was carried by the intrusion subscale $(r=-.30, p=.006)$ and was not statistically significant for the avoidance subscale, $(r=-.13, p=.26)^{4}$.

--- insert Figure 2 about here ---

\section{Correlations between tasks and comparison among studies}

In light of the unexpected finding that learning performance on the SCCT predicted more intrusions, and not fewer, it is unsurprising that spatial configuration learning and memory contextualization scores were statistically unrelated, all $r$ s being smaller than -.13, $p s>.27$. Since the present SCCT findings appeared to be in conflict with prior findings (Meyer, Smeets, Giesbrecht, Quaedflieg, Girardelli, et al., 2013), we compared the two 
datasets to explore potential explanations for this disparity. Combining the two samples, the correlation between spatial configuration learning performance and intrusions was close to zero, $r(163)=.04, p=.59$. However, the effect size difference between the two studies was substantial and statistically unlikely under the assumption that there are no systematic differences among the studies that moderated the effects (Cohen's $q=.62, z=3.87, p<.001$ ). Meanwhile, the associations between the convergent variables intrusions (diary) and intrusion symptoms (IES) did not differ significantly among studies (Cohen's $q=.17, z=1.08, p=.14$ ), combined $r(163)=.68, p<.001$.

The two studied samples did not differ significantly in mean age, $t(161)=0.2, p=.87$, gender ratio $\chi^{2}(\mathrm{df}=1)=.02, p=.89$, mean number of intrusions, intrusion-related distress, or IES scores, $t \mathrm{~s}<1.58, p \mathrm{~s}>.11$. Moreover, the participants did not differ significantly in SCCT learning performance overall, $F(1,161)=0.30, p=.58$, or in performance across epochs, $F(2,322)=1.70, p=.19$. While there were no differences in pre- and post-film NA levels or NA increase, $F \mathrm{~s}(1,160)<2.34, p \mathrm{~s}>.12$, participants in the earlier study had higher PA levels $(M=25.4, S E=0.5)$ than those in the current study $(M=22.6, S E=0.5 ; F(1,160)=12.56, p=.001$, $\left.\eta p^{2}=.07\right)$ and PA tended to decrease more in response to film viewing, though only at trendsignificant level $F(1,160)=2.83, p=.094, \eta p^{2}=.02$. However, in both samples combined, multiple regression analyses on intrusions $(d f=3,158)$ showed that spatial configuration learning did not interact significantly with mean PA levels, $\beta=.08, t=0.97, p=.33$, or with PA change, $\beta=-.05, t=-0.60, p=.55$. Thus, the explanation for the contradictory result cannot be found in terms of any moderating effects of the variables that were included in these studies.

\section{Discussion}

This study explored the association of implicit spatial configuration learning and contextualization in visual recognition memory with the development of involuntary memories of a trauma film. Implicit spatial configuration learning was, contrary to our 
predictions and earlier findings, associated with more intrusions. Meanwhile, participants who were faster at recognizing objects that were presented against their original encoding background reported fewer intrusive memories.

In our visual recognition memory task, participants were more accurate and faster in recognizing neutral and aversive objects against their original encoding background than against a different background, replicating prior findings (Van Ast et al., 2013; Van Ast, Cornelisse, Meeter, \& Kindt, 2014). Strikingly, participants who were faster in recognizing objects when presented against their original encoding background were also less prone to develop intrusions from a trauma film. Thus, the ability to memorize visual details in a bound manner along with their original visuospatial context may protect against intrusions. These findings are in line with theoretical models that ascribe a critical role to visuospatial contextual memory in the development of traumatic intrusions (e.g., Brewin et al., 2010; Ehlers, 2010).

Interestingly, only the memory contextualization effect in RT was robustly associated with intrusions, intrusion-related distress, and retrospective IES intrusion symptoms. No such effects were observed for visuospatial contextualization in discrimination performance, suggesting a dissociation between accuracy and speed of responding in our task. Hypothetically, RT might be more sensitive than accuracy to the visuospatial contextualization effects that are involved in the development of intrusions. That is, the likelihood of an intrusion might primarily depend on the ease (i.e., the speed) with which trauma-related cues can activate trauma memories in visuospatial contexts that are entirely unrelated, regardless of whether the individual is able to accurately recognize the cues. Alternatively, a possible ceiling effect in discrimination performance may have overshadowed possible associations due to a restricted score range. Supporting this interpretation, we observed no main or interaction effects of valence on recognition accuracy, unlike prior 
studies that used similar memory task with word stimuli (Van Ast et al., 2013; Van Ast et al., 2014), and that had longer intervals between the encoding and recognition phases.

Another salient outcome was that our findings did not replicate the results from a previous study (Meyer et al., 2013) that indicated a negative relationship between spatial configuration learning and intrusions. Instead, now a positive association was found. The large difference in effect size and paradoxical direction of the effects indicates that the two studies may have differed on unknown moderating variables. Both studies found highly specific effects on image-based intrusions and no associations with thought-based intrusions or other symptoms. Indeed, thought- and image-based intrusions are dissociable phenomena (Hagenaars, Brewin, van Minnen, Holmes, \& Hoogduin, 2010). Therefore, this pattern of findings suggests that configuration learning has a relationship with intrusions with a perceptual component, but that thus far unknown moderators exist that may alter the directionality of the effect.

In that respect, a few procedural differences between the current and the prior study are noteworthy. For instance, unlike Meyer et al. (2013), we applied a chin rest during trauma film viewing to reduce distraction and mobility. Also, unlike in the previous study, experimenters refrained from reassuring participants that some of the shown scenes were of educational nature, unless participants requested this information. While increased immobility may have led to more dissociative experiences during film viewing (Hagenaars et al., 2008), receiving outcome information about the film fragments may have influenced cognitive reappraisal (see Meyer et al., 2014; Meyer, Smeets, Giesbrecht, \& Merckelbach, 2012). These factors may have influenced psychophysiological stress responses (Chou, La Marca, Steptoe, \& Brewin, 2014), which may moderate the effects of stress on spatial configuration learning (Meyer, Smeets, Giesbrecht, Quaedflieg, \& Merckelbach, 2013), or directly affect intrusion levels (e.g., Krans, Pearson, Maier, \& Moulds, 2015). Interestingly, intrusions have been 
suggested to result from an interaction between the provided information and the efficiency of specific memory functions that could be altered during dissociative states (Brewin \& Burgess, 2014; Brewin et al., 2010; see also Dorahy, Peck, \& Huntjens, 2016). Therefore, an interesting route for future research would be to test whether spatial configuration learning is differentially involved in intrusive memories, depending on dissociative responses, the availability of (positive) conceptual outcome information, and psychophysiological effects of stress.

Several limitations of the present study deserve to be mentioned. Importantly, we studied visual memory contextualization and configuration learning only in relation to intrusions and avoidance symptoms. Future studies may also want to establish whether the effects are specific to involuntary memories, or to certain subtypes in a spectrum from everyday mind-wandering to highly stressful traumatic flashbacks (Meyer, Otgaar, \& Smeets, 2015). It is also important to note that we used an analogue design mimicking real-life traumatic experiences in a healthy and relatively high functioning sample. Therefore, it remains to be seen whether our findings translate directly to trauma-exposed clinical populations. Finally, our study design was not experimental and, therefore, causal relations could not be tested. It will be necessary to elucidate causal links between the specific memory functions and intrusive memory in future studies.

\section{Clinical considerations and conclusion}

Supporting models of PTSD, our results indicate that the vulnerability to develop intrusions may be related to weaker visual contextualization of trauma memories. Interestingly, scholars have also pointed to a lack of contextual memory in terms of verbal and conceptual elaboration (e.g., see Ehlers \& Clark, 2000), and studies have shown that semantic contextual information can also increase intrusions (Krans, 2013; Pearson, Ross, \& Webster, 2011) if it alters the idiosyncratic meaning of a traumatic memory in a negative way 
(for discussion, see also Brewin \& Burgess, 2014). Our results complement this literature by showing the importance of visual contextualization - and this memory function may or may not be independent of verbal or conceptual elaboration. Therefore, it may be insightful for future research to address the relationship between visuospatial and conceptual contextualization of the visual scenes.

Furthermore, the present study indicates that spatial configuration learning is unlikely to be associated with lower intrusion levels in a straightforward manner, which warrants further investigation and careful exploration of moderating variables such as dissociation, or the availability of normalizing conceptual contextual information about the trauma film. Finally, our data suggest that visual memory contextualization does not depend on efficient memory for visuospatial patterns per se, but reflects the ability to bind memories to their original visuospatial context and to transform similar experiences or events into discrete, nonoverlapping memory representations. Importantly, impairments in this ability have been proposed to underlie the overgeneralization of fear often seen in anxiety disorders, including PTSD, and may therefore represent an endophenotype for these disorders (Kheirbek, Klemenhagen, Sahay, \& Hen, 2012).

\section{Acknowledgements}

We are especially thankful to Ann-Sophie Nassal and Mma Yeebo-Agoe for their help in collecting the data. This study was supported in part by Grant 056-25-011 from the Netherlands Organization for Scientific Research (NWO) awarded to Dr. Tom Smeets. The authors declare no conflict of interest. 


\section{References}

American Psychiatric Association. (2013). Diagnostic and statistical manual of mental disorders (5th ed.). Arlington, VA: American Psychiatric Publishing.

Bennett, I. J., Barnes, K. A., Howard, J. H., \& Howard, D. V. (2009). An abbreviated implicit spatial context learning task that yields greater learning. Behavior Research Methods, 41, 391-395. doi: 10.3758/brm.41.2.391

Bonanno, G. A., \& Mancini, A. D. (2008). The human capacity to thrive in the face of potential trauma. Pediatrics, 121, 369-375. doi: 10.1542/peds.2007-1648

Bradley, M. M., \& Lang, P. J. (1999). Affective norms for English words (ANEW): Instruction manual and affective ratings. Gainesville, FL: Center for Research in Psychophysiology, University of Florida.

Brewin, C. R., \& Burgess, N. (2014). Contextualisation in the revised dual representation theory of PTSD: A response to Pearson and colleagues. Journal of Behavior Therapy and Experimental Psychiatry, 45, 217-219. doi: 10.1016/j.jbtep.2013.07.011

Brewin, C. R., Dalgleish, T., \& Joseph, S. (1996). A dual representation theory of posttraumatic stress disorder. Psychological Review, 103, 670-686.

Brewin, C. R., Gregory, J. D., Lipton, M., \& Burgess, N. (2010). Intrusive images in psychological disorders: Characteristics, neural mechanisms, and treatment implications. Psychological Review, 117, 210-232. doi: 10.1037/a0018113

Chou, C.-Y., La Marca, R., Steptoe, A., \& Brewin, C. R. (2014). Biological responses to trauma and the development of intrusive memories: An analog study with the trauma film paradigm. Biological Psychology, 103, 135-143. doi:

10.1016/j.biopsycho.2014.08.002 
Craik, F. I. M., \& Lockhart, R. S. (1972). Levels of processing: A framework for memory research. Journal of Verbal Learning and Verbal Behavior, 11, 671-684. doi: $10.1016 / \mathrm{S} 0022-5371(72) 80001-\mathrm{X}$

Darves-Bornoz, J. M., Alonso, J., de Girolamo, G., Graaf, R. d., Haro, J. M., Kovess-Masfety, V., .. . Vilagut, G. (2008). Main traumatic events in Europe: PTSD in the European study of the epidemiology of mental disorders survey. Journal of Traumatic Stress, 21, 455-462. doi: 10.1002/jts.20357

Dorahy, M. J., Peck, R. K., \& Huntjens, R. J. C. (2016). The impact of dissociation on perceptual priming and intrusions after listening to auditory narratives. Journal of Trauma \& Dissociation, null-null. doi: 10.1080/15299732.2015.1134746

Ehlers, A. (2010). Understanding and treating unwanted trauma memories in posttraumatic stress disorder. Zeitschrift Fur Psychologie-Journal of Psychology, 218, 141-145. doi: $10.1027 / 0044-3409 / \mathrm{a} 000021$

Ehlers, A., \& Clark, D. M. (2000). A cognitive model of posttraumatic stress disorder. Behaviour Research and Therapy, 38, 319-345. doi: 10.1016/S0005-7967(99)00123-0

Eichenbaum, H. (2004). Hippocampus: Cognitive processes and neural representations that underlie declarative memory. Neuron, 44, 109-120. doi: 10.1016/j.neuron.2004.08.028

Farrell, S. (2012). Temporal clustering and sequencing in short-term memory and episodic memory. Psychological Review, 119, 223.

Fyhn, M., Hafting, T., Treves, A., Moser, M.-B., \& Moser, E. I. (2007). Hippocampal remapping and grid realignment in entorhinal cortex. Nature, 446, 190-194.

Goldfarb, Elizabeth V., Chun, Marvin M., \& Phelps, Elizabeth A. (2016). Memory-Guided Attention: Independent Contributions of the Hippocampus and Striatum. Neuron, 89, 317-324. doi: 10.1016/j.neuron.2015.12.014 
Hagenaars, M. A., Brewin, C. R., van Minnen, A., Holmes, E. A., \& Hoogduin, K. A. L. (2010). Intrusive images and intrusive thoughts as different phenomena: Two experimental studies. Memory, 18, 76-84. doi: 10.1080/09658210903476522

Hagenaars, M. A., van Minnen, A., Holmes, E. A., Brewin, C. R., \& Hoogduin, K. A. L. (2008). The effect of hypnotically induced somatoform dissociation on the development of intrusions after an aversive film. Cognition \& Emotion, 22, 944-963. doi: 10.1080/02699930701575151

Holmes, E. A., James, E. L., Coode-Bate, T., \& Deeprose, C. (2009). Can playing the computer game "Tetris" reduce the build-up of flashbacks for trauma? A proposal from cognitive science. Plos One, 4, 6. doi: e415310.1371/journal.pone.0004153

Horowitz, M. J., Wilner, N., \& Alvares, W. (1979). Impact of Event Scale: A measure of subjective stress. Psychosomatic Medicine, 41, 209-218.

Kheirbek, M. A., Klemenhagen, K. C., Sahay, A., \& Hen, R. (2012). Neurogenesis and generalization: A new approach to stratify and treat anxiety disorders. Nature neuroscience, 15, 1613-1620. doi: 10.1038/nn.3262

Krans, J. (2013). The self and involuntary memory: Identifying with the victim increases memory accessibility for stressful events. Consciousness and cognition, 22, 12981304. doi: 10.1016/j.concog.2013.08.006

Krans, J., Pearson, D. G., Maier, B., \& Moulds, M. L. (2015). Contextual representations of negative images modulate intrusion frequency in an intrusion provocation paradigm. Journal of Behavior Therapy and Experimental Psychiatry. doi: 10.1016/j.jbtep.2015.09.004

Lang, P. J., Bradley, M. M., \& Cuthbert, B. N. (2005). International Affective Picture System (IAPS): Instruction Manual and Affective Ratings. Technical Report A-6. Gainesville, FL: University of Florida. 
Lee, I. A., \& Preacher, K. J. (2013, September). Calculation for the test of the difference between two dependent correlations with one variable in common. Retrieved from http://quantpsy.org

Lovibond, S. H., \& Lovibond, P. F. (1995). Manual for the Depression Anxiety Stress Scales (2nd ed.). Sidney: Psychology Foundation.

Manns, J. R., \& Squire, L. R. (2001). Perceptual learning, awareness, and the hippocampus. Hippocampus, 11, 776-782. doi: 10.1002/hipo.1093

Meyer, T., Otgaar, H., \& Smeets, T. (2015). Flashbacks, intrusions, mind-wandering Instances of an involuntary memory spectrum: A commentary on Takarangi, Strange, and Lindsay (2014). Consciousness and Cognition, 33, 24-29. doi: 10.1016/j.concog.2014.11.012

Meyer, T., Quaedflieg, C. W. E. M., Giesbrecht, T., Meijer, E., Abiad, S., \& Smeets, T. (2014). Frontal EEG asymmetry as predictor of physiological responses to aversive memories. Psychophysiology, 51, 853-865. doi: 10.1111/psyp.12230

Meyer, T., Smeets, T., Giesbrecht, T., \& Merckelbach, H. (2012). The efficiency of reappraisal and expressive suppression in regulating everyday affective experiences. Psychiatry Research, 200, 964-969. doi: 10.1016/j.psychres.2012.05.034

Meyer, T., Smeets, T., Giesbrecht, T., Quaedflieg, C. W. E. M., Girardelli, M. M., Mackay, G. R. N., \& Merckelbach, H. (2013). Individual differences in spatial configuration learning predict the occurrence of intrusive memories. Cognitive, Affective, \& Behavioral Neuroscience, 13, 186-196. doi: 10.3758/s13415-012-0123-9

Meyer, T., Smeets, T., Giesbrecht, T., Quaedflieg, C. W. E. M., \& Merckelbach, H. (2013). Acute stress differentially affects spatial configuration learning in high and low cortisol-responding healthy adults. European Journal of Psychotraumatology, 4. doi: 10.3402/ejpt.v4i0.19854 
O'Reilly, R. C., \& Rudy, J. W. (2000). Computational principles of learning in the neocortex and hippocampus. Hippocampus, 10, 389-397. doi: 10.1002/10981063(2000)10:4<389::AID-HIPO5>3.0.CO;2-P

Pearson, D. G., Ross, F. D. C., \& Webster, V. L. (2011). The importance of context: Evidence that contextual representations increase intrusive memories. Journal of Behavior Therapy and Experimental Psychiatry, 43, 573-580.

Preston, A. R., \& Gabrieli, J. D. E. (2008). Dissociation between explicit memory and configural memory in the human medial temporal lobe. Cerebral Cortex, 18, 21922207. doi: 10.1093/cercor/bhm245

Rivest, L. P. (1994). Statistical properties of Winsorized means for skewed distributions. Biometrika, 81, 373-383. doi: 10.1093/biomet/81.2.373

Rubin, D. C., Berntsen, D., \& Bohni, M. K. (2008). Memory-based model of Posttraumatic Stress Disorder: Evaluating basic assumptions underlying the PTSD diagnosis. Psychological Review, 115, 985-1011. doi: 10.1037/a0013397

Stanislaw, H., \& Todorov, N. (1999). Calculation of signal detection theory measures. Behavior Research Methods, Instruments, \& Computers, 31, 137-149. doi: 10.3758/BF03207704

Staresina, B. P., \& Davachi, L. (2010). Object unitization and associative memory formation are supported by distinct brain regions. The Journal of Neuroscience, 30, 9890-9897. doi: 10.1523/JNEUROSCI.0826-10.2010

Steiger, J. H. (1980). Tests for comparing elements of a correlation matrix. Psychological Bulletin, 87, 245-251.

Van Ast, V. A., Cornelisse, S., Meeter, M., Joëls, M., \& Kindt, M. (2013). Time-dependent effects of cortisol on the contextualization of emotional memories. Biological Psychiatry, 74, 809-816. doi: 10.1016/j.biopsych.2013.06.022 
Van Ast, V. A., Cornelisse, S., Meeter, M., \& Kindt, M. (2014). Cortisol mediates the effects of stress on the contextual dependency of memories. Psychoneuroendocrinology, 41, 97-110. doi: 10.1016/j.psyneuen.2013.12.007

Watson, D., Clark, L. A., \& Tellegen, A. (1988). Development and validation of brief measures of positive and negative affect: The PANAS scales. Journal of Personality and Social Psychology, 54, 1063-1070. 


\section{Footnotes}

${ }^{1}$ We established that the background pictures were emotionally neutral in a pilot study with 10 participants providing SAM ratings for valence $(M=5.6 ; S D=0.6 ; 1=$ extremely unpleasant; $9=$ extremely pleasant $)$ and arousal $(M=2.7 ; S D=1.3 ; 1=$ low arousal; $9=$ intense arousal). The SAM ratings from the current study confirmed that neutral trials were rated higher on valence $(M=6.2, S D=1.1)$ and lower on arousal $(M=2, S D=1.1)$ than negative trials (valence: $M=3.2, S D=1$; arousal: $M=4.5, S D=1.7)$, $t \mathrm{~s}(80)>13, p s<.001$.

${ }^{2}$ NA increase correlated with higher levels of intrusions $(r=.28, p=.011)$, intrusionrelated distress $(r=.42, p<.001)$, and IES scores $(r=.35, \mathrm{p}=.001)$. Also DASS-21 scores tended to correlate with intrusions $(r=.19, p=.09)$ and IES scores $(r=.22, p=.054)$. Therefore, for each correlation analysis reported below, we also ran multiple linear regressions entering NA increase and DASS-21 scores first, and the predictors of interest (i.e., spatial configuration or context-dependence scores) second. This approach did not change the conclusions of the correlation analyses reported below.

${ }^{3}$ Similar to Meyer, Smeets, Giesbrecht, Quaedflieg, Girardelli, et al. (2013), we found non-sphericity in the array by epoch interaction (Mauchly's $W=.84, \chi^{2}=6.7, p=.036$ ), indicating that some participants may have quickly reached a ceiling in learning, whereas others continued to improve across epochs. In line with this, participants with below-median spatial configuration learning scores in the first epoch improved across epochs, $F(2,78)=39.82, \varepsilon=.81, p<.001, \eta p^{2}=.51$, whereas participants with above-median scores did not, $F(2,80)=1.18, \varepsilon=.86, p=.31, \eta p^{2}=.03$.

${ }^{4}$ For exploration, we repeated the correlation analyses separately for RT memory contextualization in neutral and negative trials, revealing consistent associations with PTSD analogue symptoms in terms of direction. Although most effects were descriptively larger for RT memory contextualization in negative versus neutral trials, there were no statistically 
significant differences according to Steiger's (1980) test of equality of dependent correlations, all $z s<1.41, p s>.16$ (Lee \& Preacher, 2013, September). Furthermore, we found that these findings uniquely pertained to contextualization; similar analyses for the RT differences between old and new trials and between negative and neutral trials did not reveal any significant relationship with any of the intrusion measures, all $p s>.18$. 
Table 1.

Reported PTSD analogue symptoms $(N=81)$

\begin{tabular}{llc}
\hline Method & Indicator & $M(S D)$ \\
\hline Diary & All memories & $4.1(4.2)$ \\
& With image component & $2.9(3.0)$ \\
& With thought component & $2.5(3.6)$ \\
& Distress & $3.7(2.5)$ \\
IES & Re-experiencing & $7.2(5.2)$ \\
& Avoidance & $4.9(4.9)$ \\
& Total PTSD scores & $12.1(9.0)$ \\
\hline
\end{tabular}

Note. IES=Impact of Event Scale. 


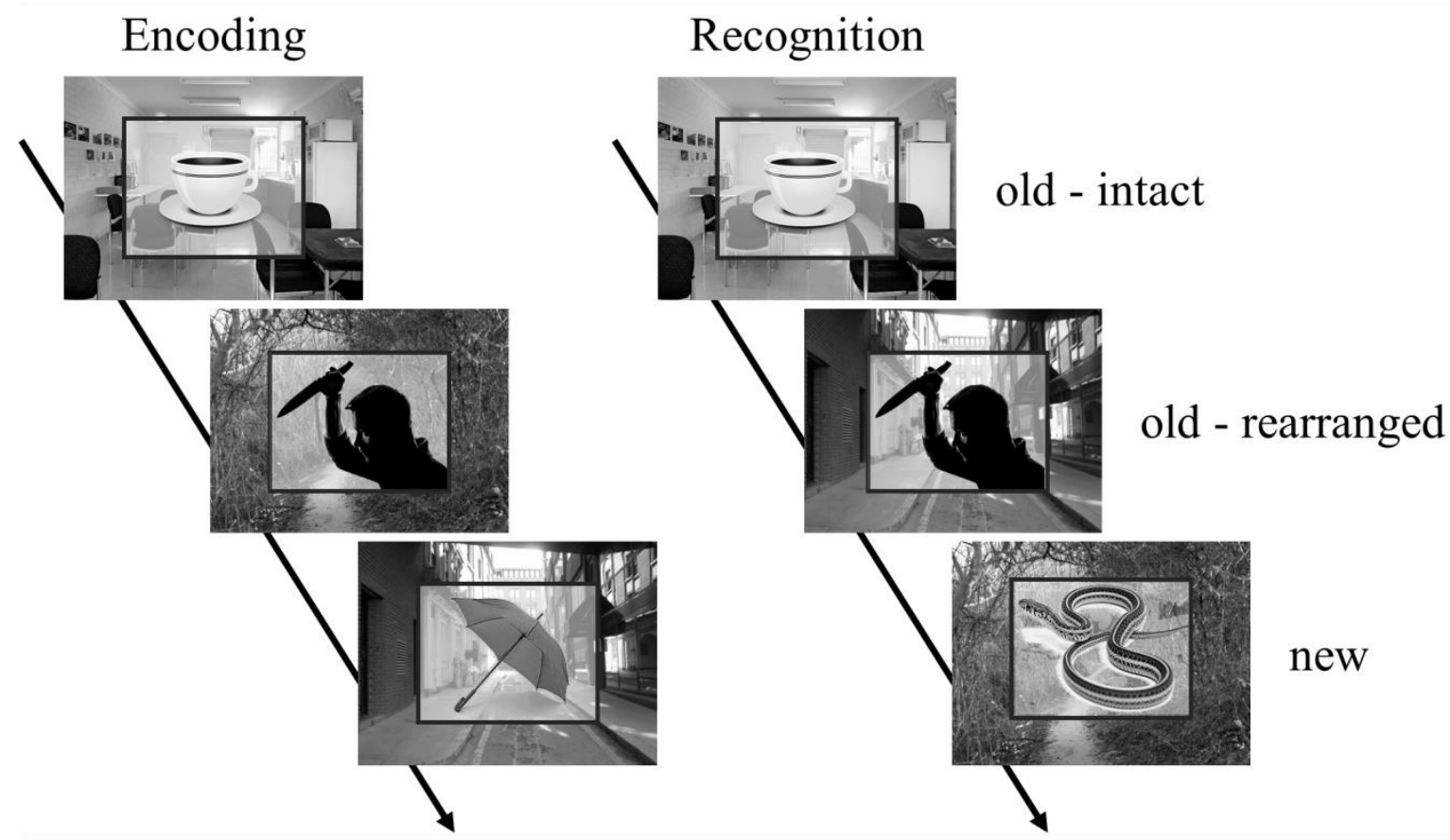

Figure 1. Visual recognition memory task: Encoding phase and conditions of the recognition phase. This figure serves as an illustration and does not depict actual objects taken from the IAPS. Parts of this figure are protected by creative-commons licenses (for details, please contact the corresponding author). 


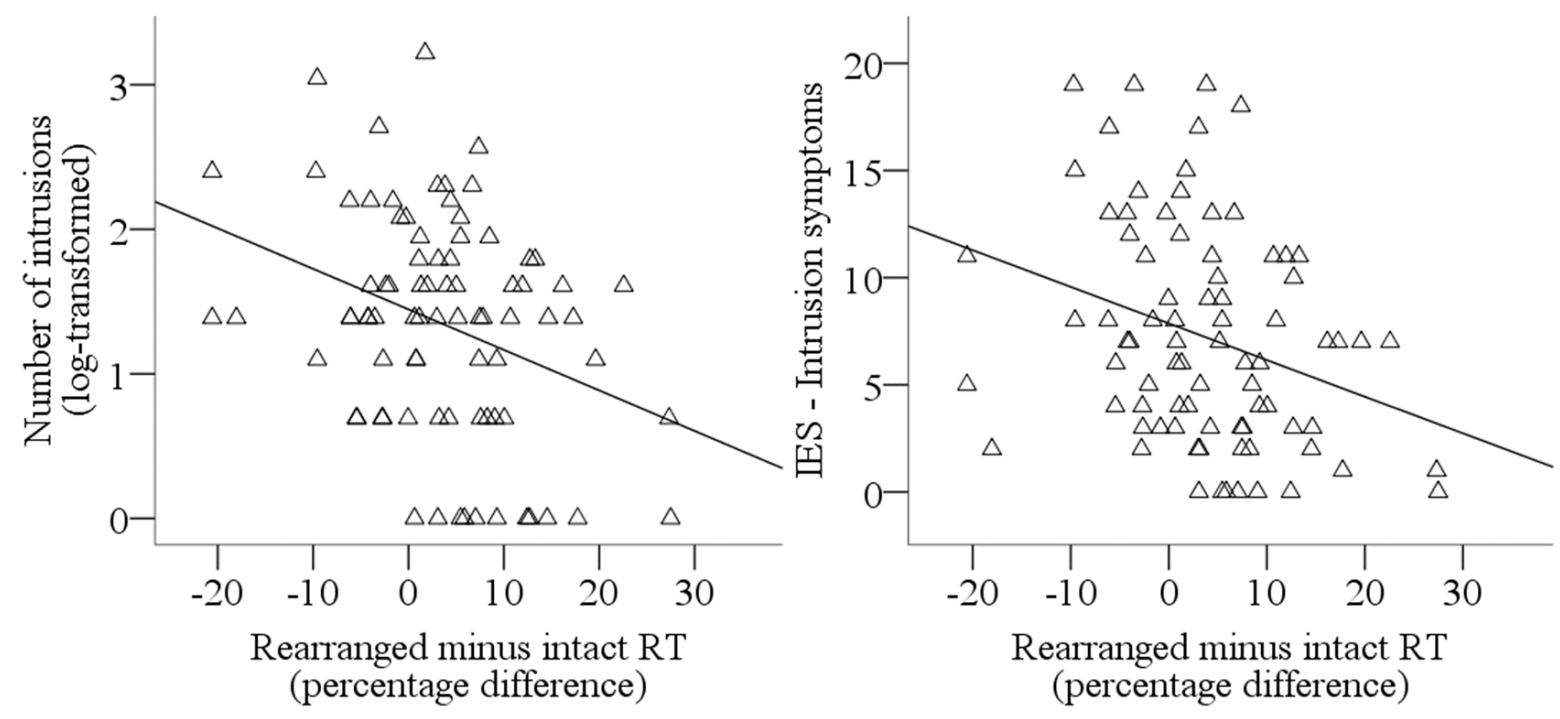

Figure 2. Scatterplots depicting the correlations between the reaction time (RT) advantage in intact versus rearranged trials (i.e., memory contextualization scores; percentage difference corrected for baseline RT) with intrusion symptoms, i.e. the total number of intrusions in the diary in the left graph, and scores on the IES intrusion subscale on the right. 